\title{
Impact of different exercise interventions on anxiety and depression in breast cancer patients
}

\author{
DOI: https://doi.org/10.5114/pq.2019.87737
}

\author{
Tetiana Odynets ${ }^{1}$, Yuriy Briskin ${ }^{2}$, Valentina Todorova ${ }^{3}$, Olha Bondarenko ${ }^{3}$ \\ ${ }_{1}$ Department of Physical Rehabilitation, Khortytsia National Academy, Zaporizhzhya, Ukraine \\ 2 Department of Theory of Sport and Physical Culture, Lviv State University of Physical Culture, Lviv, Ukraine \\ ${ }^{3}$ Department of Gymnastics and Martial Arts, South Ukrainian National Pedagogical University named after K.D. Ushynsky, \\ Odessa, Ukraine
}

\section{Abstract}

Introduction. The aim of the study was to examine the impact of different exercise interventions on anxiety and depression in breast cancer patients through 1-year outpatient rehabilitation.

Methods. Overall, 138 breast cancer patients were assessed for eligibility. After excluding 14 women, 124 patients were randomly allocated to water exercise interventions (group A, $n=50$ ), Pilates exercise interventions (group B, $n=44$ ), and yoga exercise interventions (group C, $n=30$ ). Finally, 115 patients completed relevant interventions and were analysed. The participants reported anxiety and depression using the Hospital Anxiety and Depression Scale at baseline and after 6 and 12 months of the interventions.

Results. After 12 months, a decrease in anxiety and depression was observed by $5.64(p<0.001)$ and $4.09(p<0.001)$ points in group A, by $5.75(p<0.001)$ and $4.60(p<0.001)$ points in group $B$, and by $4.07(p<0.001)$ and $3.14(p<0.001)$ points in group $C$, respectively. Group A showed significantly better results than group $C$ in anxiety (by 1.54 points; $p<0.01$ ) and depression (by 1.40 points; $p<0.05$ ). Group $B$ obtained significantly better results than group $C$ in anxiety (by 1.61 points; $p<0.01$ ) and depression (by 1.81 points; $p<0.01$ ).

Conclusions. Practising a water exercise program resulted in a better decrease of anxiety and depression levels compared with Pilates and yoga interventions.

Key words: anxiety, depression, breast cancer, psychological distress

\section{Introduction}

Breast cancer is the most frequently diagnosed cancer among women worldwide and often leads to mental health disorders and lower quality of life. Numerous reports have highlighted that many breast cancer patients experience cancerrelated fear of recurrence, emotional distress, negative mood symptoms, anxiety, post-traumatic stress disorder, depression after completing treatment [1-5]. Breast cancer diagnosis, performed surgical intervention and adjuvant treatment, changed body image and hair loss, as well as fear about partner's acceptance result in anxiety disorders and depressive reactions $[6,7]$.

Therefore, it is important to address anxiety and depression because they can create certain difficulties in performing daily activities and also significantly impair the patients' quality of life.

Physical activity is increasingly recognized as an effective method of reducing emotional distress and improving life quality among patients who survived cancer. A growing body of research indicates the benefits of yoga exercises [8-14], cognitive behavioural therapy [15], mindfulness-based stress reduction [16, 17], physical activity [18], music therapy [19, 20], progressive muscular relaxation [21-22], and mixed aerobic and muscle strengthening exercise [23] for improving mental and physical conditions in breast cancer patients.
Research data suggest that yoga exercises are effective tools for relieving fatigue and arm pain, and improving sleep disturbances and fitness outcomes in cancer patients $[10,11,14,24]$, but the problem of the effect of yoga on reducing anxiety and depression in breast cancer survivors is still not fully resolved. A study by Cramer et al. [13] indicates evidence for yoga as a supportive intervention for reducing fatigue and sleep disturbances and improving life quality. Moreover, the authors emphasize that yoga can be as effective as other exercise interventions, which, however, requires further investigation. A randomized controlled trial performed by Taso et al. [25] showed that an 8-week yoga intervention was effective only for reducing fatigue, not for depression or anxiety in breast cancer patients.

The relevance of this study is underlined by the lack of data regarding Pilates and water exercises, whose role in reducing anxiety and depression in breast cancer survivors has not been sufficiently investigated. Despite the available publications, there are no studies in the literature that would identify the most effective program for reducing anxiety and depression in breast cancer survivors.

Consequently, the issue of comparing different interventions and identifying the most effective one in outpatient rehabilitation for breast cancer patients becomes relevant.

Correspondence address: Tetiana Odynets, Department of Theory of Sport and Physical Culture, Lviv State University of Physical Culture, 11 Kostiushko Street, Lviv 79000, Ukraine, e-mail: tatyana01121985@gmail.com

Received: 12.07 .2019

Accepted: 14.08.2019

Citation: Odynets T, Briskin Y, Todorova V, Bondarenko O. Impact of different exercise interventions on anxiety and depression in breast cancer patients. Physiother Quart. 2019;27(4):31-36; doi: https://doi.org/10.5114/pq.2019.87737. 


\section{Subjects and methods}

\section{Participants}

Comprehensive sociodemographic characteristics of the analysed patients are shown in Table 1. There were no baseline differences between the studied groups.
A CONSORT flow diagram of the study is presented in Figure 1. A total of 138 breast cancer patients were assessed for eligibility. After excluding 14 women owing to stage 3 of cancer, chronic obstructive lung disease, metastases, ischaemic heart disease, or refusal to participate, 124 patients were randomly allocated to water exercise intervention (group $A$, $n=50$ ), Pilates exercise intervention (group $B, n=44$ ), and

Table 1. Sociodemographic and treatment-related characteristics of the analysed participants

\begin{tabular}{|c|c|c|c|c|}
\hline \multirow{2}{*}{\multicolumn{2}{|c|}{ Characteristics }} & \multicolumn{3}{|c|}{ Groups } \\
\hline & & $\mathrm{A}(n=45)$ & $\mathrm{B}(n=40)$ & $C(n=30)$ \\
\hline \multicolumn{2}{|l|}{ Age $(M \pm S D)$} & $58.84 \pm 1.36$ & $59.40 \pm 1.24$ & $59.10 \pm 1.37$ \\
\hline \multirow{2}{*}{ Race } & White, $n(\%)$ & 44 (98\%) & 38 (95\%) & $29(97 \%)$ \\
\hline & Black, $n(\%)$ & $1(2 \%)$ & $2(5 \%)$ & $1(3 \%)$ \\
\hline \multicolumn{2}{|c|}{ Married/committed relationship, $n$ (\%) } & $42(94 \%)$ & $36(90 \%)$ & $28(93 \%)$ \\
\hline \multicolumn{2}{|c|}{ High school graduates, $n$ (\%) } & $21(47 \%)$ & $20(50 \%)$ & $16(53)$ \\
\hline \multicolumn{2}{|c|}{ College graduates, $n(\%)$} & $20(44 \%)$ & $18(45 \%)$ & $13(43 \%)$ \\
\hline \multicolumn{2}{|c|}{ High school graduates, $n(\%)$} & $4(9 \%)$ & $2(5 \%)$ & $1(3 \%)$ \\
\hline \multicolumn{2}{|c|}{ Body mass index $\left(\mathrm{kg} / \mathrm{m}^{2}\right)(M \pm S D)$} & $24.12 \pm 0.42$ & $24.20 \pm 0.44$ & $24.11 \pm 0.38$ \\
\hline \multirow{2}{*}{ Treatment } & Radiotherapy, $n$ (\%) & 42 (94\%) & 38 (95\%) & 27 (90\%) \\
\hline & Chemotherapy, $n$ (\%) & $3(6 \%)$ & $2(5 \%)$ & $3(10 \%)$ \\
\hline \multicolumn{2}{|c|}{ Mastectomy by Madden, $n$ (\%) } & 45 (100\%) & $40(100 \%)$ & $30(100 \%)$ \\
\hline \multicolumn{2}{|c|}{ Time since treatment completion (months) $(M \pm S D)$} & $5.12 \pm 2.54$ & $5.13 \pm 2.79$ & $5.13 \pm 2.87$ \\
\hline \multirow{2}{*}{ Cancer stage } & Stage $1, n(\%)$ & $15(33 \%)$ & $14(35 \%)$ & $9(30 \%)$ \\
\hline & Stage $2, n(\%)$ & $30(67 \%)$ & $26(65 \%)$ & $21(70 \%)$ \\
\hline
\end{tabular}

$M-$ mean, $S D$ - standard deviation

Figure 1. A CONSORT flow diagram of the study

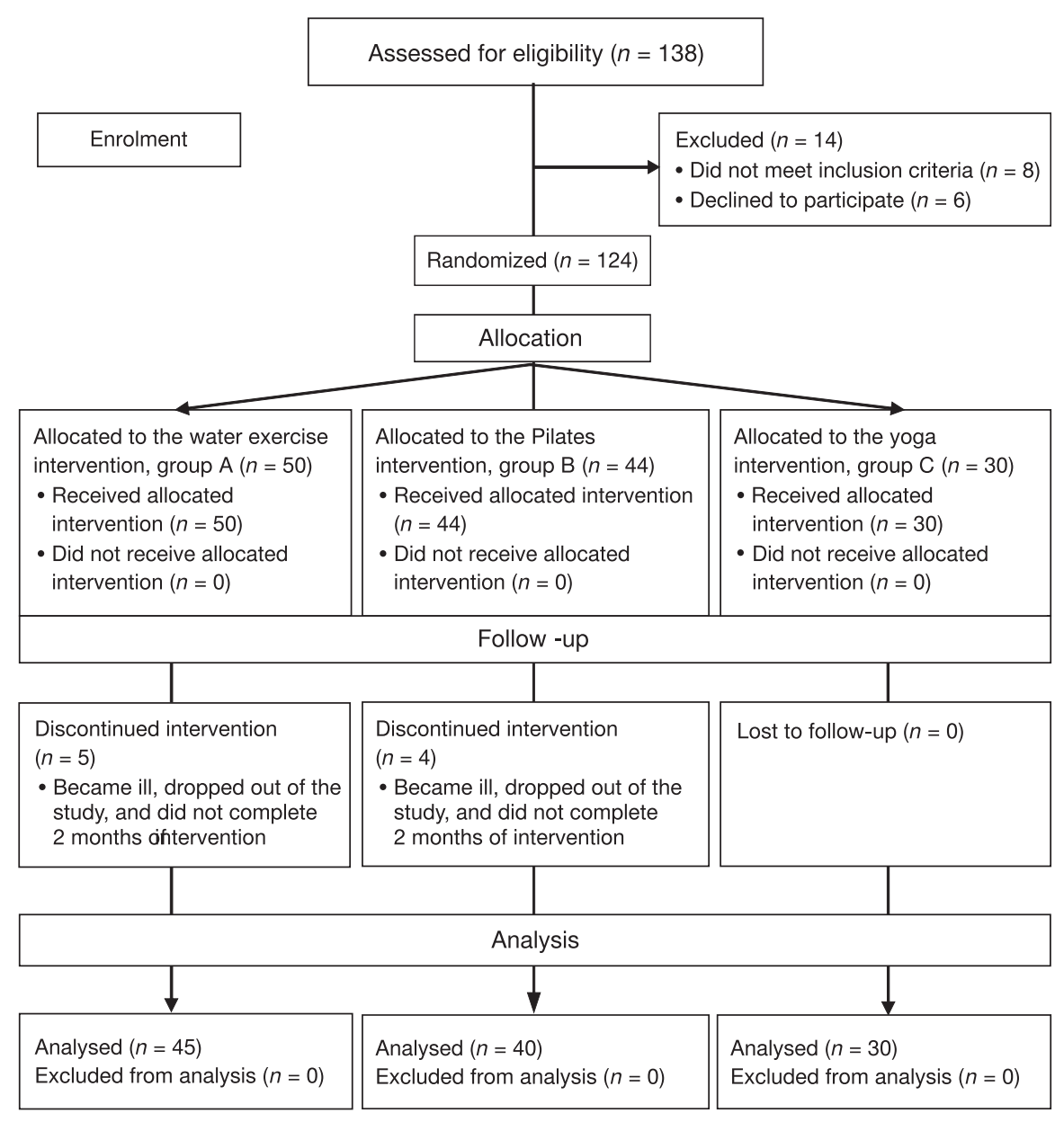


yoga exercise intervention (group C, $n=30$ ). Finally, after dropping out, 115 patients completed relevant interventions and were analysed. The participants reported anxiety and depression using the Hospital Anxiety and Depression Scale at baseline and after 6 and 12 months of the interventions. The focus on women aged $50-60$ years was applied because the incidence of breast cancer is the highest in this age category.

\section{Interventions}

The components of the 3 interventions were based on the patients' individual baseline functional status of the cardiovascular system, which was classified as average, lower than average, or low level. The groups were engaged in the proposed interventions 3 times a week for 1 hour through 1 year of outpatient rehabilitation. The groups had the same time limit and were conducted by the same physiotherapist.

Water exercise intervention for women of group A was built on a rational combination of swimming, breathing exercises (static, dynamic), combined developing exercises, and exercises of local impact on different muscle groups using various initial positions: standing, half-squatting, sitting, lying on noodles. Noodles, blades, and water dumbbells were applied to increase the load. The intensity of physical activity depended on the motion status and varied from $45 \%$ to $70 \%$ of heart rate reserve.

Group B received Pilates exercises 3 times per week for 12 months. The aggregated time of Pilates also depended on the functional status of the cardiovascular system and lasted from 40-45 minutes at the adaptation rehabilitation stage to 1 hour at the training stage. The intensity varied from $40-45 \%$ to $60-70 \%$ of heart rate reserve. All exercises were performed with musical accompaniment and clearly showed by the physiotherapist.

The most important part of a Pilates session for women was Pilates matwork at the adaptation and training stages. It was necessary for the acquisition of initial Pilates skills and principles by breast cancer patients and also for successful task solution. Flexibility and resistance exercises were directed at the muscles of the scapular waist, lower limbs, back, and abdomen.

Group C attended 3 yoga exercise sessions per week for 12 months. The proposed yoga exercise program for breast cancer patients also differed depending on the duration and intensity of the session, the number of exercise repetitions, as well as their percentage. The training of yoga physical exercises began with the study of the asanas technique and breathing exercises; after successful assimilation, the static and motor activity gradually proceeded to the implementation of integral dynamic asanas complexes, which were performed without a pause of rest. The breathing exercises were performed with an emphasis on long exhalation and were well coordinated with movements. The duration of asana retention, its complexity, and the number of repetitions depended on the level of the functional status of the woman and gradually increased as the patient's body adapted to the load. The intensity of yoga exercise varied from low to moderate. The participants constantly concentrated on their own feelings while doing the exercises. For successful training in respiratory exercises, mainly stable sedentary poses were used that allowed to maintain a comfortable position for a long time and contributed to the greatest concentration of attention on the involved muscles in the act of breathing.

\section{Measurement}

Hospital Anxiety and Depression Scale [26] was used to identify the presence and severity of anxiety and depression. This reliable and informative questionnaire consists of 14 questions divided into 2 subscales ( 7 for anxiety, 7 for depression). The results of self-analysis were classified as follows: 0-7 points: no reliably expressed symptoms of anxiety or depression, 8-10 points: subclinical anxiety/depression, 11 points or more: clinically expressed anxiety/depression.

\section{Statistical analysis}

The data recorded (mean and standard error of the mean) were analysed by using the Statistica for Windows software (version 8.00). Before the concluding analysis, data were evaluated for normality assumption, homogeneity, and occurrence of extreme scores. The distribution of the recorded data was tested with the Shapiro-Wilk test. Such analysis was performed as a preliminary measure before parametric calculations of the analysis of difference. The dependent $t$-test was applied to analyse anxiety and depression parameters in each group between baseline and post-intervention. The independent sample $t$-tests served to compare post-intervention results and identify the most effective interventions.

\section{Ethical approval}

The research related to human use has complied with all the relevant national regulations and institutional policies, has followed the tenets of the Declaration of Helsinki, and has been approved by the review board of the Khortytsia National Academy.

\section{Informed consent}

Informed consent has been obtained from all individuals included in this study.

\section{Results}

Changes in the Hospital Anxiety and Depression Scale parameters in breast cancer patients through 1 year of outpatient rehabilitation are shown in Table 2.

Table 2. Hospital Anxiety and Depression Scale parameters in breast cancer patients through 1 year of outpatient rehabilitation

\begin{tabular}{|l|c|c|c|}
\hline \multirow{2}{*}{ Indicator } & \multicolumn{3}{|c|}{$\begin{array}{c}(M \pm m) \\
\text { Hospital Anxiety and Depression Scale points }\end{array}$} \\
\cline { 2 - 4 } & At baseline & 6 months & 12 months \\
\cline { 2 - 4 } & & \multicolumn{3}{|c|}{ Post-intervention } \\
\hline Group A $(n=45)$ & $11.06 \pm 0.47$ & $7.77 \pm 0.40^{*}$ & $5.42 \pm 0.39^{*}$ \\
\hline Anxiety & $8.55 \pm 0.44$ & $6.17 \pm 0.38^{*}$ & $4.46 \pm 0.35^{*}$ \\
\hline Depression & $11.10 \pm 0.39$ & $7.62 \pm 0.40^{*}$ & $5.35 \pm 0.39^{*}$ \\
\hline Group B $(n=40)$ & $8.65 \pm 0.37$ & $5.65 \pm 0.41^{*}$ & $4.05 \pm 0.39^{*}$ \\
\hline Anxiety & $11.03 \pm 0.50$ & $8.10 \pm 0.47^{*}$ & $6.96 \pm 0.42^{*}$ \\
\hline Depression & $9.00 \pm 0.64$ & $6.56 \pm 0.52^{*}$ & $5.86 \pm 0.46^{*}$ \\
\hline Group C $(n=30)$ & & \\
\hline Anxiety & & & \\
\hline Depression & & & \\
\hline
\end{tabular}

$M-$ mean, $m$ - error of mean

${ }^{*} p<0.001$ compared with the baseline data 
Table 3. Comparison of Hospital Anxiety and Depression Scale parameters among the studied groups after 6 months of outpatient rehabilitation

\begin{tabular}{|l|c|c|c|c|c|c|}
\hline \multirow{2}{*}{ Indicator } & \multicolumn{2}{|c|}{ Hospital Anxiety and Depression Scale points $(M \pm m)$ after 6 months } & \multirow{2}{*}{$p_{1}$} & \multirow{2}{*}{$p_{2}$} & \multirow{2}{*}{$p_{3}$} \\
\cline { 2 - 4 } & Group A & Group B & Group C & & \\
\hline Anxiety & $7.77 \pm 0.40$ & $7.62 \pm 0.40$ & $8.10 \pm 0.47$ & $>0.05$ & $>0.05$ & $>0.05$ \\
\hline Depression & $6.17 \pm 0.38$ & $5.65 \pm 0.41$ & $6.56 \pm 0.52$ & $>0.05$ & $>0.05$ & $>0.05$ \\
\hline
\end{tabular}

$M$ - mean, $m$ - error of mean, $p_{1}$-comparison between groups $\mathrm{A}$ and $\mathrm{B}, p_{2}$ - comparison between groups $\mathrm{A}$ and $\mathrm{C}$, $p_{3}$ - comparison between groups $\mathrm{B}$ and $\mathrm{C}$

Table 4. Comparison of Hospital Anxiety and Depression Scale parameters among the studied groups after 1 year of outpatient rehabilitation

\begin{tabular}{|l|c|c|c|c|c|c|}
\hline \multirow{2}{*}{ Indicator } & \multicolumn{2}{|c|}{ Hospital Anxiety and Depression Scale points $(M \pm m)$ after 12 months } & \multirow{2}{*}{$p_{1}$} & \multirow{2}{*}{$p_{2}$} & \multirow{2}{*}{$p_{3}$} \\
\cline { 2 - 5 } & Group A & Group B & Group C & & \\
\hline Anxiety & $5.42 \pm 0.39$ & $5.35 \pm 0.39$ & $6.96 \pm 0.42$ & $>0.05$ & $<0.01$ & $<0.01$ \\
\hline Depression & $4.46 \pm 0.35$ & $4.05 \pm 0.39$ & $5.86 \pm 0.46$ & $>0.05$ & $<0.05$ & $<0.01$ \\
\hline
\end{tabular}

$M$ - mean, $m$ - error of mean, $p_{1}$ - comparison between groups $\mathrm{A}$ and $\mathrm{B}, p_{2}$ - comparison between groups $\mathrm{A}$ and $\mathrm{C}$, $p_{3}$ - comparison between groups $\mathrm{B}$ and $\mathrm{C}$

On the basis of the results of a 6-month monitoring, it was established that different exercise interventions contributed to a significant improvement in the emotional state of the breast cancer patients.

There was a decrease in the indicators of anxiety and depression by $3.29(p<0.001)$ and $2.38(p<0.001)$ points in the first half of the year, and by $5.64(p<0.001)$ and 4.09 $(p<0.001)$ points after 1 year, respectively, in group A. A similar situation was observed for Pilates and yoga interventions during 1 year of outpatient rehabilitation.

After 6 months, there was a decrease in the anxiety and depression level by $3.48(p<0.001)$ and $3.00(p<0.001)$ points in the group performing Pilates, and by $2.93(p<0.001)$ and $2.44(p<0.001)$ points, respectively, in the group performing yoga.

After 12 months, a decrease was found in the anxiety and depression level by $5.75(p<0.001)$ and $4.60(p<0.001)$ points in group B, and by $4.07(p<0.001)$ and $3.14(p<0.001)$ points, respectively, in group $\mathrm{C}$.

A comparison of the results obtained after 6 months between the groups revealed that there were no statistically significant differences (Table 3).

When comparing the results of the assessment of anxiety and depression between groups $A$ and $B$ after a year, no statistically significant differences were observed, but a comparison with group $C$ revealed such differences. On the basis of the results after 12 months (Table 4), women of group A showed significantly better outcomes than those in group C: by 1.54 points $(p<0.01)$ for anxiety and by 1.40 points $(p<0.05)$ for depression. After 12 months of Pilates training, women of group B had significantly better results compared with group C: by 1.61 points $(p<0.01)$ for anxiety and by 1.81 points $(p<0.01)$ for depression. In general, the beneficial effect of water exercises on the anxiety and depression level was established compared with Pilates and yoga interventions.

A detailed analysis of the Hospital Anxiety and Depression Scale parameters after a year of outpatient rehabilitation showed that $85 \%$ of women in group A, $87 \%$ in group $\mathrm{B}$, and $75 \%$ in group $\mathrm{C}$ did not present anxiety at all. Subclinical manifestations of anxiety were observed in $15 \%$ patients of group A, $13 \%$ of group B, and $20 \%$ of group C.

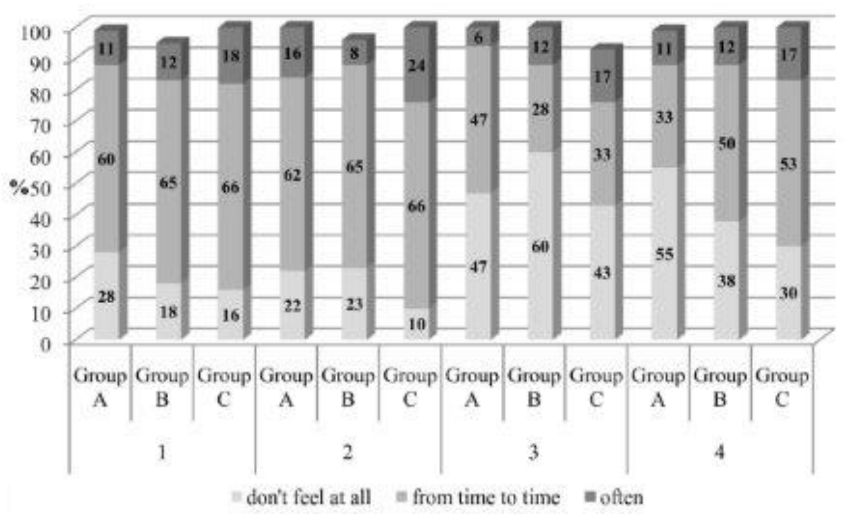

1 - I feel stress, 2 - Worrying thoughts go through my mind,

3 - I feel tense, 4 - I get sudden feelings of panic

Figure 2. The patients' responses regarding anxiety after a year of intervention

patients in group C. Details for the women's responses regarding anxiety after a year of outpatient rehabilitation are presented in Figure 2.

A detailed analysis of the responses to the statement 'I feel stress' indicated stress occurrence from time to time in $60 \%$ of group $A$, while in group $B$ and $C$ it was recorded in $65 \%$ and $66 \%$ of patients, respectively.

The analysis of the responses to the statement 'Worrying thoughts go through my mind' showed that only $16 \%$ of women in group $A, 8 \%$ in group $B$, and $24 \%$ in group $C$ often experienced this feeling; worrying thoughts appeared from time to time in $62 \%, 65 \%$, and $66 \%$ of the respective groups. Only $11 \%$ of respondents in group $A, 12 \%$ in group $B$, and $17 \%$ in group $\mathrm{C}$ totally agreed with the statement 'I get sudden feelings of panic'; panic was experienced from time to time by $33 \%, 35 \%$, and $53 \%$ of women, respectively.

A detailed analysis of women's responses regarding depression after a year of outpatient rehabilitation is presented in Figure 3. The responses to the statement 'I still enjoy the things I used to enjoy' showed that $47 \%$ of women in group $A$, $58 \%$ in group $B$, and $33 \%$ in group $C$ totally agreed with this statement; the feeling was sometimes present in $40 \%, 35 \%$, and $60 \%$, rarely in $13 \%, 7 \%$, and $7 \%$ of respondents, respectively. The results of the participants' responses revealed that 


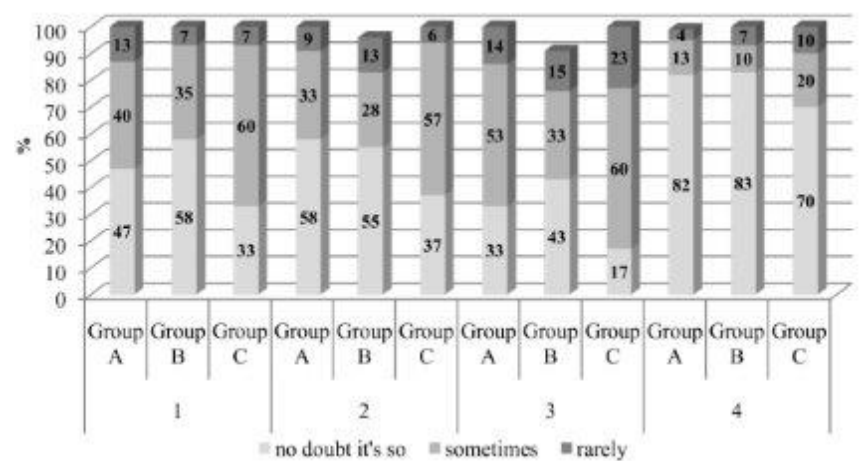

1 - I still enjoy the things I used to enjoy, 2 - I can laugh and see the funny side of things, $3-$ I feel cheerful, $4-$ I can enjoy a good book, radio or TV program

Figure 3. The patients' responses regarding depression after a year of intervention

$58 \%$ of women in group A, $55 \%$ in group B, and $37 \%$ in group $\mathrm{C}$ could laugh and see the funny side of things. Overall, $82 \%$ of women in group A, $83 \%$ in group B, and $70 \%$ in group $\mathrm{C}$ were satisfied with a good book or a radio or TV program; $13 \%, 10 \%$, and $20 \%$, respectively, partially agreed with this statement.

\section{Discussion}

The results from the current study convincingly demonstrate that applying different interventions throughout a year of outpatient rehabilitation significantly improved the emotional state of breast cancer patients.

The assessment of the Hospital Anxiety and Depression Scale parameters after a year of outpatient rehabilitation revealed that water exercise intervention was a more valuable and favourable tool for reducing the anxiety and depression level than Pilates or yoga interventions in breast cancer women.

The results agree with recent studies in that breast cancer patients have negative mood symptoms, anxiety, and depression after cancer treatment [5, 8, 13, 17, 27], and systematic physical exercises are considered as effective tools for enhancing the emotional state and life quality of the women $[18,24]$.

Previous studies $[5,8,11,25]$ have primarily focused on the effects of yoga on reducing fatigue, depression, and stress in breast cancer patients, but different procedures, duration, and intensity of intervention produce some obstacles in comparing the previous results with the context of the present research

A study by Włoch et al. [23] evidenced that muscle strengthening interventions performed during 12-13 weeks, twice a week, contributed to reducing markers of depression and cognitive function.

In the current study, the actual level of the breast cancer patients' cardiovascular system functional status was taken into account in the proposed different interventions. The general structure and content of the interventions for patients varied in the duration of the asanas, intensity, and number of repetitions. The combination of various special exercises, breathing exercises, calisthenic routine, and relaxation modes had a great impact on anxiety and depression in the breast cancer patients.

The findings obtained in the current study indicate benefits of water exercises for reducing stress and negative mood symptoms in breast cancer patients.

\section{Limitations}

The limitation of our study was that the anxiety and depression level was measured with a self-reported questionnaire, and differences in the emotional condition of women could have an impact on the obtained results. Further studies are needed to investigate the effectiveness of different interventions on anxiety and depression in heterogeneous populations.

\section{Conclusions}

Practising a water exercise program resulted in a better decrease of the anxiety and depression level compared with Pilates and yoga interventions.

\section{Disclosure statement}

No author has any financial interest or received any financial benefit from this research.

\section{Conflict of interest}

The authors state no conflict of interest.

\section{References}

1. Odynets T, Briskin Y, Sydorko O. Psycho-emotional state and quality of life characteristics in women with postmastectomy syndrome with different types of attitude to the disease. Physiother Quart. 2018;26(1):9-12; doi: 10.5114/pq.2018.74706.

2. Yi JC, Syrjala KL. Anxiety and depression in cancer survivors. Med Clin North Am. 2017;101(6):1099-1113; doi: 10.1016/j.mcna.2017.06.005.

3. Merckaert I, Lewis F, Delevallez F, Herman S, Caillier M, Delvaux N, et. al. Improving anxiety regulation in patients with breast cancer at the beginning of the survivorship period: a randomized clinical trial comparing the benefits of single-component and multiple-component group interventions. Psychooncology. 2017;26(8):1147-1154; doi: 10.1002/pon.4294.

4. Carlson LE, Tamagawa R, Stephen J, Drysdale E, Zhong L, Speca M. Randomized-controlled trial of mindfulness-based cancer recovery versus supportive expressive group therapy among distressed breast cancer survivors (MINDSET): long-term follow-up results. Psychooncology. 2016;25(7):750-759; doi: 10.1002/pon.4150.

5. Zuo X-L, Li Q, Gao F, Yang L, Meng F-J. Effects of yoga on negative emotions in patients with breast cancer: a meta-analysis of randomized controlled trials. Int $\mathrm{J}$ Nurs Sci. 2016;3(3):299-306; doi: 10.1016/j.ijnss.2016. 07.009 .

6. Brandt-Salmeri A, Przybyła-Basista H. Depression and marital satisfaction: the mediating role of sexual satisfaction and perceived partner acceptance in women after mastectomy. Health Psychol Rep. 2019;7(2):121132; doi: 10.5114/hpr.2019.84213.

7. Sowa M, Głowacka-Mrotek I, Monastyrska E, Nowikiewicz T, Mackiewicz-Milewska M, Hagner W, et al. Assessment of quality of life in women five years after breast cancer surgery, members of Breast Cancer Self-Help Groups - non-randomized, cross-sectional study. Contemp Oncol. 2018;22(1):20-26; doi: 10.5114/wo.2018. 74389.

8. Cramer $\mathrm{H}$, Lauche R, Klose $\mathrm{P}$, Lange $\mathrm{S}$, Langhorst J, Dobos GJ. Yoga for improving health-related quality of life, mental health and cancer-related symptoms in women diagnosed with breast cancer. Cochrane Database 
Syst Rev. 2017;1:CD010802; doi: 10.1002/14651858. CD010802.pub2.

9. Dangi AA, Aurangabadkar SK, Deo MV. Effect of a structured yoga program on fatigue, depression, cardiorespiratory fitness, and quality of life in a postmenopausal breast cancer survivor. Int J Yoga. 2018;11(3):255257; doi: 10.4103/ijoy.IJOY_61_17.

10. Hughes DC, Darby N, Gonzalez K, Boggess T, Morris RM, Ramirez AG. Effect of a six-month yoga exercise intervention on fitness outcomes for breast cancer survivors. Physiother Theory Pract. 2015;31(7):451-460; doi: 10.3109/09593985.2015.1037409.

11. McCall M. Yoga intervention may improve health-related quality of life (HRQL), fatigue, depression, anxiety and sleep in patients with breast cancer. Evid Based Nurs. 2018;21(1):9; doi: 10.1136/eb-2017-102673.

12. Danhauer SC, Addington EL, Cohen L, Sohl SJ, Van Puymbroeck M, Albinati NK, et al. Yoga for symptom management in oncology: a review of the evidence base and future directions for research. Cancer. 2019;125(12): 1979-1989; doi: 10.1002/cncr.31979.

13. Cramer H, Lange S, Klose P, Paul A, Dobos G. Yoga for breast cancer patients and survivors: a systematic review and meta-analysis. BMC Cancer. 2012;12:412; doi: 10.1186/1471-2407-12-412.

14. Vadiraja HS, Rao RM, Nagarathna R, Nagendra HR, Patil S, Diwakar RB, et al. Effects of yoga in managing fatigue in breast cancer patients: a randomized controlled trial. Indian J Palliat Care. 2017;23(3):247-252; doi: 10.4103/IJPC.IJPC_95_17.

15. Brothers BM, Yang HC, Strunk DR, Andersen BL. Cancer patients with major depressive disorder: testing a biobehavioral/cognitive behavior intervention. J Consult Clin Psychol. 2011;79(2):253-260; doi: 10.1037/a0022566.

16. Reich RR, Lengacher CA, Alinat CB, Kip KE, Paterson C, Ramesar S, et al. Mindfulness-based stress reduction in post-treatment breast cancer patients: immediate and sustained effects across multiple symptom clusters. J Pain Symptom Manage. 2017;53(1):85-95; doi: 10.1016/ j.jpainsymman.2016.08.005.

17. Stan DL, Collins NM, Olsen MM, Croghan I, Pruthi S. The evolution of mindfulness-based physical interventions in breast cancer survivors. Evid Based Complement Alternat Med. 2012;2012:758641; doi: 10.1155/2012/ 758641.

18. Zhu G, Zhang X, Wang $Y$, Xiong $H$, Zhao $Y$, Sun F. Effects of exercise intervention in breast cancer survivors: a metaanalysis of 33 randomized controlled trails. Onco Targets Ther. 2016;9:2153-2168; doi: 10.2147/OTT.S97864.

19. Palmer JB, Lane D, Mayo D, Schluchter M, Leeming R. Effects of music therapy on anesthesia requirements and anxiety in women undergoing ambulatory breast surgery for cancer diagnosis and treatment: a randomized controlled trial. J Clin Oncol. 2015;33(28):31623168; doi: 10.1200/JCO.2014.59.6049.

20. Li XM, Zhou KN, Yan H, Wang DL, Zhang YP. Effects of music therapy on anxiety of patients with breast cancer after radical mastectomy: a randomized clinical trial. J Adv Nurs. 2012;68(5):1145-1155; doi: 10.1111/j.13652648.2011.05824.x.

21. Yilmaz SG, Arslan S. Effects of progressive relaxation exercises on anxiety and comfort of Turkish breast cancer patients receiving chemotherapy. Asian Pac J Cancer Prev. 2015;16(1):217-220; doi: 10.7314/apjcp.2015.16. 1.217.
22. Prystupa E, Odynets T, Briskin Y, Tyshchenko V. Effects of an individualised physical rehabilitation intervention enhanced by progressive muscular relaxation and visualisation exercises on psycho-emotional state in women after breast cancer surgery. Physiother Pract Res. 2019;40(1):21-27; doi: 10.3233/PPR-180121.

23. Włoch A, Bocian A, Biskup M, Krupnik S, Opuchlik A, Ridan T, et al. Effectiveness of specific types of structured physical activities in the rehabilitation of post-mastectomy women: a systematic review. Med Stud Stud Med. 2018;34(1):86-92; doi: 10.5114/ms.2018.74826.

24. Pelekasis P, Matsouka I, Koumarianou A. Progressive muscle relaxation as a supportive intervention for cancer patients undergoing chemotherapy: a systematic review. Palliat Support Care. 2017;15(4):465-473; doi: 10.1017/S1478951516000870.

25. Taso CJ, Lin HS, Lin WL, Chen SM, Huang WT, Chen SW. The effect of yoga exercise on improving depression, anxiety, and fatigue in women with breast cancer: a randomized controlled trial. J Nurs Res. 2014;22(3):155164; doi: 10.1097/jnr.0000000000000044.

26. Zigmond AS, Snaith RP. The hospital anxiety and depression scale. Acta Psychiatr Scand. 1983;67(6):361370; doi: 10.1111/j.1600-0447.1983.tb09716.x.

27. Fradelos EC, Latsou D, Mitsi D, Tsaras K, Lekka D, Lavdaniti $M$, et al. Assessment of the relation between religiosity, mental health, and psychological resilience in breast cancer patients. Contemp Oncol. 2018;22(3): 172-177; doi: 10.5114/wo.2018.78947. 